\title{
REVIEW
}

\section{Right ventricular myocardial stiffness and relaxation components by kinematic model-based analysis}

\author{
Yasunobu Hayabuchi, MD, PhD \\ Department of Pediatrics, Department of Pediatrics and community medicine, Tokushima University, Tokushima, Japan
}

\begin{abstract}
Right ventricle (RV) has frequently been described as the forgotten ventricle in the circulation. However, its importance in various cardiac diseases is now unquestioned. This recognition has led to improved risk stratification and development of algorithms for intervention, which incorporate measurements of RV function as key components of the assessment of many conditions. The diastolic function plays an important role in determining ventricular filling and stroke volume. Abnormal left ventricular (LV) diastolic function has been recognized in many cardiovascular diseases and is associated with worse outcomes, including total mortality and hospitalizations due to heart failure. In this review, we define what global RV diastolic function is, and how to measure it. This article indicates the validation of kinematic model parameters for assessing RV diastolic function. J. Med. Invest. $67: 11-20$, February, 2020
\end{abstract}

Keywords : right ventricle, diastolic function, stiffness, relaxation, pressure phase plane

\section{INTRODUCTION}

Systemic hypertension is recognized as one of the major causes of diastolic dysfunction in the left ventricle (LV) (1). However, there is limited knowledge regarding the effect of chronic pressure overload on the right ventricular (RV) diastolic function. Since the assessment of the RV diastolic function is challenging $(2,3)$, Patients with pulmonary arterial hypertension (PAH) ultimately develop right heart failure (4). Previous studies have demonstrated that PAH patients have reduced systolic function as measured by the right ventricular (RV) ejection fraction. Although most clinical research has focused on systolic function, normal RV filling is also essential to maintain exercise activity and adapt to acute and chronic overload. However, knowledge of the role of RV diastolic function in PAH is limited (3-5). Abnormalities in both active cross-bridge relaxation and passive elastic recoil are observed in the hypertrophied RV myocardium, eventually leading to RV diastolic dysfunction, which results in increases in RV filling and right atrial pressures. Indeed, they are associated with disease progression and increased mortality in both adults and children with PAH (5-7). However, assessment of RV diastolic function is challenging (3-5). Consequently, few studies have investigated RV diastolic function, particularly in pediatric PAH patients (9). Accurate measurement of RV diastolic function could contribute to improved clinical management of these patients.

The time constant $(\tau)$ is considered the best empirical standard for estimating the rate of pressure decrease in the assessment of left ventricular (LV) diastolic function $(10,11)$. However, the pattern of RV pressure decrease is quite different from that of LV pressure decrease (12). The peak rate of pressure decrease (dP/dt_min) is not a reliable reference point for evaluating the onset of RV diastole, because it appears when the major portion of RV pressure decrease has already occurred (12). The time

Received for publication August 20, 2019 ; accepted September 6, 2019.

Address correspondence and reprint requests to Yasunobu Hayabuchi, MD, Department of Pediatrics, Department of Pediatrics and community medicine, Tokushima University, Kuramoto-cho-3, Tokushima 770-8305, Japan and Fax : +81-886-31-8697. constant $(\tau)$ evaluates a relatively much shorter segment in the RV than in the LV.

On the other hand, noninvasive assessment of diastolic function is commonly achieved through the use of pulsed Doppler echocardiography. Although conventional echocardiographic parameters are often indicative of dysfunction, their utility in characterizing the relaxation and stiffness/elastic recoil is limited (13). To overcome the limitations of these parameters, Kovács et al. quantified LV diastolic function using a mechanistic model of filling that was determined by cross-bridge uncoupling relaxation, elastic recoil/restoring forces, and initial displacement (load) (14-16).

We recently demonstrated the feasibility and usefulness of kinematic model parameters obtained from RV pressure waveform for evaluating RV diastolic function (17). Furthermore, we have shown that the physical and physiological principles govern the transtricuspid flow pattern and that a mathematical model would be able to correctly quantify the pathological RV diastolic property in children and adolescents with PAH (18).

\section{PRESSURE MEASUREMENT AND DIASTOLIC FUNC- TION}

The ventricular pressure $(\mathrm{P}(\mathrm{t}))$, the time derivatives of pressure (dP/dt), LV and RV end-diastolic pressures (LVEDP and RVEDP), maximum and minimum pressures and pressure derivatives (Pmax, Pmin, dP/dt_max, and dP/dt_min), and the IVR pressure inflection point are determined. The pressure phase plane (PPP), where $\mathrm{dP} / \mathrm{dt}$ is plotted against $\mathrm{P}(\mathrm{t})$, is delineated (Fig. 1) (19-21). LVEDP is defined by the LV pressure at the ECG R-wave peak. The mitral valve opening (MVO) and tricuspid valve opening (TVO) times are determined as the time points where the decaying pressure contours are closest to the LVEDP and RVEDP, respectively, of the subsequent filling beat $(22,23)$. 
A

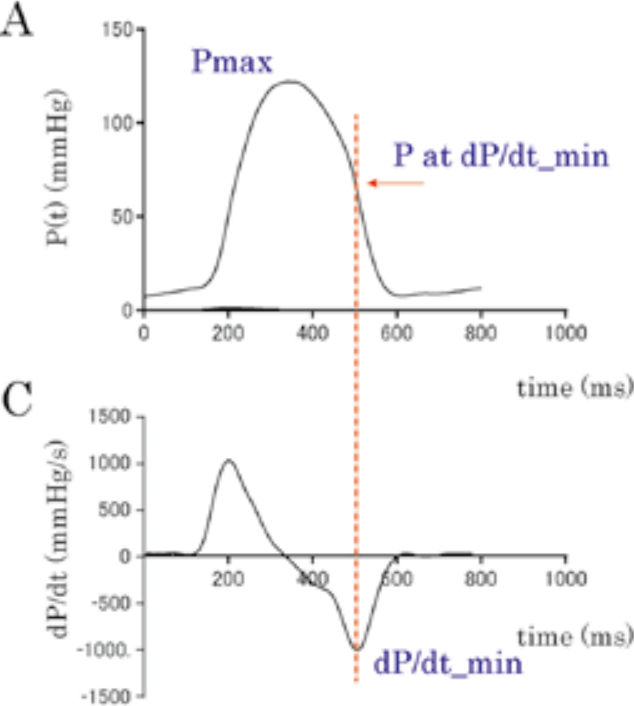

$\mathrm{E}$

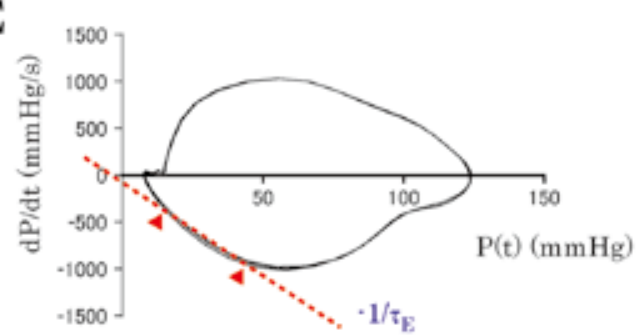

B

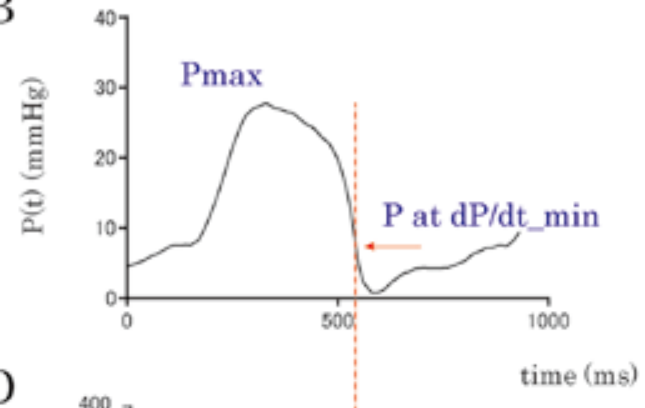

D

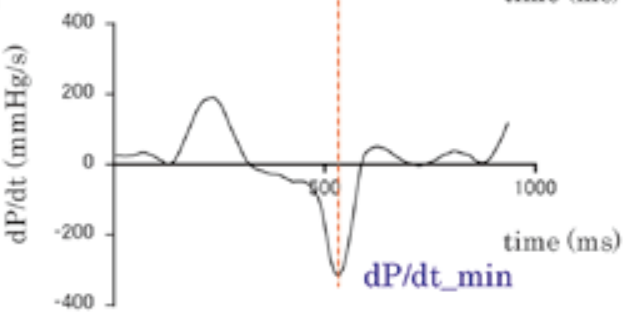

$\mathrm{F}$

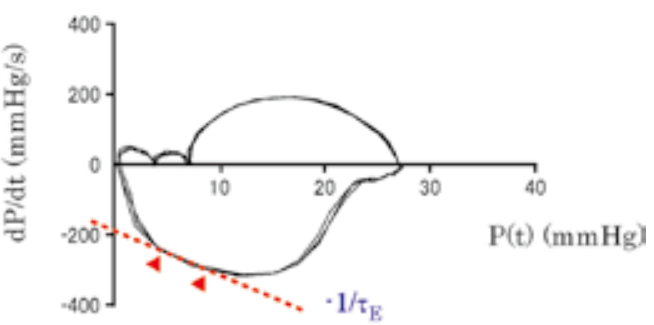

Fig. 1 Representative example of time courses of left ventricular (LV) pressure (A), right ventricular (RV) pressure (B), LV dP/ $\mathrm{dt}(\mathrm{C})$, and RV dP/dt (D). The vertical red dotted line marks the times at dP/dt_min of the LV and RV. LV dP/dt_min occurs when LV pressure falls to $54 \%$ of its peak-systolic pressure (Pmax). RV dP/dt_min occurs when RV pressure falls to $33 \%$ of Pmax. The pressure phase planes (PPPs; $\mathrm{dP} / \mathrm{dt}$ vs. P) of LV (E) and RV (F) are shown. The negative inverse of the slope of the isovolumic pressure decrease shown by the red dotted lines in the PPP indicates monoexponential $\tau$. The time interval for the calculation of the time constant is shown between the triangles. Note that the time interval of RV is much shorter than that of LV.

Figure modifies from Haybuchi Y, et al. (17) with permission.

\section{Assessment of monoexponential and logistic time constants}

Diastolic function has traditionally been evaluated using the IVR time constant, which describes the pressure decrease. In the monoexponential model of pressure decay, it is assumed that the time derivative of pressure decay is proportional to pressure. The governing differential equation for the monoexponential model is

$$
\tau_{E} \frac{d P(t)}{\mathrm{d} t}+\left(P_{0}-P_{\infty}\right) e^{-t / \tau_{E}}=0
$$

or

$$
P(t)=\left(P_{0}-P_{\infty}\right) e^{-t / \tau_{E}}+P_{\infty}
$$

where $\tau \mathrm{E}$ is the monoexponential time constant of IVR pressure, and $\mathrm{P}_{\infty}$ is the pressure asymptote $(10,11)$. A convenient way to determine it is to plot Eq.1A in the PPP, where a straight line with a slope of $-1 / \tau \mathrm{E}$ and an intercept on the $\mathrm{dP} / \mathrm{dt}$ axis is inscribed, and it is then fit to the IVR portion of the loop that is inscribed by $\mathrm{P}(\mathrm{t})$ for the cardiac cycle (Fig. 1E, F) (20, 21). However, because there are curvilinear IVR segments, a straightline fit to the IVR portion of the loop is not always physiological (Fig. 1F). In addition, RV pressure decay in particular has been shown to have curvilinear IVR segments (12). Thus, $\tau$ E may not be suitable for evaluating RV diastolic function.

Another empirical constant has been proposed as an alternative to fit these common curved IVR segments of PPP trajectories (21). The logistic time constant $\tau \mathrm{L}$, which is similar to $\tau \mathrm{E}$, provides an empirical fit, in which the rate of pressure decrease is proportional to the square of the pressure and is given by

$$
\frac{P^{2}}{P_{A}}+\tau_{L} \frac{d P}{d t}+P(t)-P_{B}=0
$$

or

$$
P(t)=\frac{P_{A}}{1+e^{-t / \tau_{L}}}+P_{B}
$$

where $\tau_{\mathrm{L}}$ is the logistic time-constant of IVR, and the pressure asymptote is given by the sum of $\mathrm{PA}_{\mathrm{A}}$ and $\mathrm{PB}$. This logistic relationship is quadratic in $\mathrm{P}(\mathrm{t})$, and it can only produce, and, therefore, best fit, curvilinear PPP IVR contours in the PPP.

The slope of the $\mathrm{dP} / \mathrm{dt}$ versus $\mathrm{P}(\mathrm{t})$ plot over the interval between $10 \mathrm{~ms}$ after $\mathrm{dP} / \mathrm{dt} \_\mathrm{min}$ and $10 \mathrm{~ms}$ before the estimated MVO time determined by the least-squares method is equal to $-1 / \tau \mathrm{E}(10,11,19)$. $\tau \mathrm{L}$ is obtained using the methods of Matsubara $e t$ $a l$. with a customized Levenberg-Marquardt algorithm $(21,24)$. 


\section{KINEMATIC MODELING OF VENTRICULAR PRES- SURE DECAY}

Chung and Kovács previously showed that LV pressure decay is accurately determined mathematically by the interactions of inertial, stiffness, and relaxation forces using physiological-kinematic arguments, and they published their experimental results (25). The relative contributions of stiffness and relaxation to IVR pressure are characterized by a stiffness parameter and a damping or relaxation parameter. $(25,26)$ In the kinematic model, IVR pressure is predicted from before $\mathrm{dP} / \mathrm{dt}$ _min to near MVO. Their theory applies the kinematics of the damping oscillator governed by the (mass normalized) equation of motion :

$$
\frac{d^{2} x}{d t^{2}}+c \frac{d x}{d t}+k x=0
$$

where $\mathrm{k}$ is stiffness and $\mathrm{c}$ is damping $(25,26)$. The parameters of this model are stiffness/restoring Ek and damping/relaxation $\mu$. The equation for LV pressure during this IVR phase is

$$
\frac{d^{2} P}{d t^{2}}+\frac{1}{\mu} \frac{d P}{d t}+E_{k}\left(P-P_{\infty}\right)=0
$$

The solution for this equation in the underdamped regime (1/ $\left.\mu^{2}<4 \mathrm{Ek}\right)$ for pressure or for the time derivative of pressure is given by

$$
\begin{aligned}
& P(t)=e^{-t / 2 \mu}\left(\frac{\dot{P}_{0}+P_{d} / 2 \mu}{\omega} \sin (\omega \cdot t)+P_{0} \cos (\omega \cdot t)\right)+P_{\infty} \\
& \frac{d P}{d t}(t)=e^{-t / 2 \mu}\left(-\left(\frac{\dot{P}_{0} / \mu+2 E_{k} P_{0}}{2 \omega}\right) \sin (\omega \cdot t)+\dot{P}_{0} \cos (\omega \cdot t)\right]
\end{aligned}
$$

where $\mathrm{Po}$ is the initial pressure assuming a zero pressure asymptote, $\mathrm{P}^{\prime} \mathrm{o}$ is the initial time derivative of pressure, and

$$
\omega=\sqrt{4 \mathrm{Ek}_{\mathrm{k}}-1 / \mu^{2}} / 2
$$

The critically damped $\left(1 / \mu^{2}=4 \mathrm{Ek}\right)$ and overdamped $\left(1 / \mu^{2}>4 \mathrm{Ek}\right)$ solutions are provided by evaluating Eqs. 4 and 5 at the $\omega=0$ (critically damped) or $\omega=i \beta$ (overdamped) limits.

When $1 / \mu^{2}=4 \mathrm{Ek}$ (critically damped kinematics) :

$$
P(t)=P_{0} e^{-t / 2 \mu}+\left(\dot{P}_{0}+P_{0} / 2 \mu\right) e^{-t / 2 \mu} t+P_{\infty}
$$

When $1 / \mu^{2}>4 \mathrm{Ek}$ (overdamped kinematics) :

$$
\begin{aligned}
& P(t)=e^{-t / 2 \mu}\left(\frac{\dot{P}_{0}+P_{0} / 2 \mu}{\beta} \sinh (\beta \cdot t)+P_{0} \cosh (\beta \cdot t)\right)+P_{\infty} \\
& \beta=\sqrt{1 / \mu^{2}-4 \mathrm{Ek}} / 2
\end{aligned}
$$

To extract Ek and $\mu$ from an isovolumic pressure contour, which is the equivalent to solving the "inverse problem of IVR pressure," the procedure is as follows.

With a custom-automated Java program (Pressure Decay Analysis Tokushima (PDA-Tokushima) ver. 1.05) (17), hemodynamic data were analyzed (Fig. 2). Ek, $\mu, \mathrm{Po}$, and $\mathrm{P}^{\prime} \mathrm{o}$ were extracted for each IVR pressure contour in each subject via Eq. 5 from $\mathrm{dP} / \mathrm{dt}$ versus $\mathrm{t}$ data by a Levenberg-Marquardt fitting algorithm to the $\mathrm{dP} / \mathrm{dt}$ data $(17,25-27)$. The initial point for the fitting is from the inflection point in the IVR pressure contour before $\mathrm{dP} / \mathrm{dt}$ _min, while the endpoint was taken to be $10 \mathrm{~ms}$ prior to the estimated MVO or TVO time. Having found Ek, $\mu, \mathrm{Po}$, and $\mathrm{P}^{\prime}$, Eq. 4 is used to determine $\mathrm{P}$ with the Levenberg-Marquardt algorithm and the other 4 parameters held constant. Since IVR pressure contours are non-physiological and noisy, they generate high root mean square error (RMSE) values between the raw

A

B
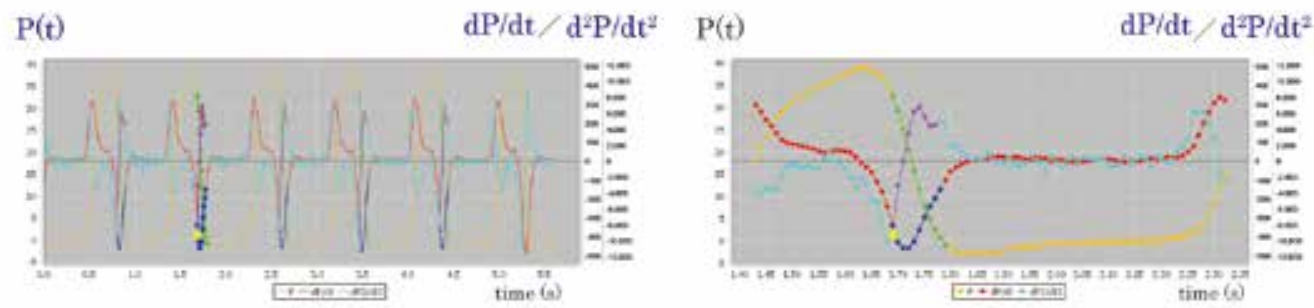

C

D
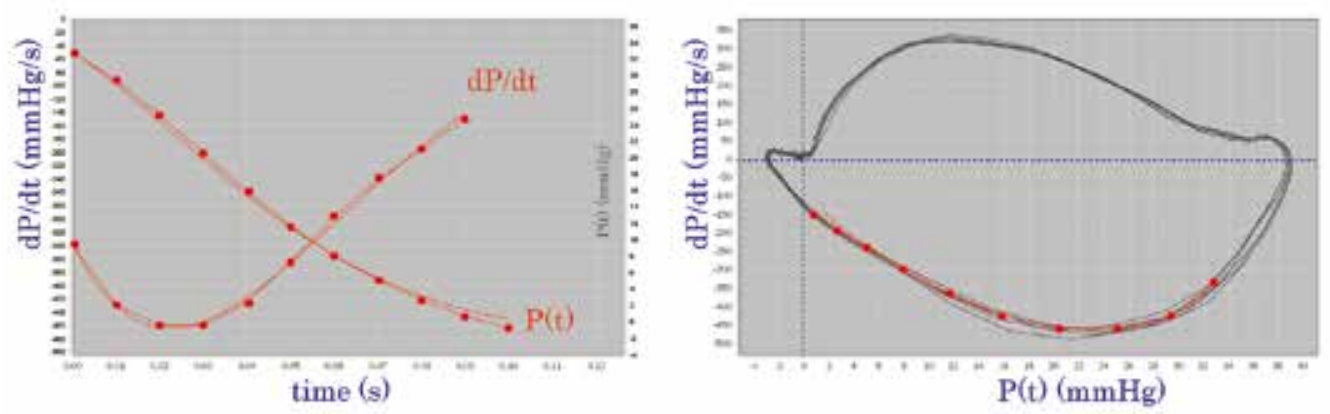

Fig. 2 Appearance of the custom-automated Java program (Pressure Decay Analysis Tokushima (PDA-Tokushima) ver. 1.05) for the calculation of the kinematic model parameters.

Pressure, $\mathrm{dP} / \mathrm{dt}$, and $\mathrm{d}_{2} \mathrm{P} / \mathrm{dt}_{2}$ of several cardiac cycles are shown (A). One cardiac cycle of these waves is shown as an enlarged display (B). The actual measured pressure and dP/dt are shown as dots, and fitting curves for $\mathrm{P}$ and $\mathrm{dP} / \mathrm{dt}$ obtained from equations 4 and 5 are shown as the curvilinear lines (C). PPP trajectories are shown by the black curvilinear portion. The actual measurements are shown by the red dots. The calculated kinematic model fitting curve using Ek and $\mu$ is shown by the red curvilinear portion (D).

Figure modifies from Haybuchi Y, et al. (17) with permission. 
$\mathrm{dP} / \mathrm{dt}$ data and the model fit $\mathrm{dP} / \mathrm{dt}$ when they are compared with acceptable physiological data. Therefore, beats having the largest 50th percentile RMSE values are to be discarded. Thus, only physiological smooth data are included in the final analysis, and this had the additional advantage of being automated, which minimized observer bias in beat selection.

\section{RIGHT AND LEFT VENTRICULAR PRESSURE DE- CREASES IN NORMAL SUBJECTS}

Representative examples of cardiac cycles in the control group are shown as LV and RV pressures, $\mathrm{dP} / \mathrm{dt}$ time courses, and PPP (Fig. 1). The pattern and rate of RV pressure decay can be compared with those of LV pressure decay. Both ventricles show two distinct phases of pressure decrease, an initial accelerative phase and a subsequent decelerative phase separated by the corresponding dP/dt_min. In the LV, the initial accelerative phase (until LV-dP/dt_min) encompasses $25.05 \% \pm 3.1 \%$, while the major part of the LV pressure decreases during the subsequent phase in a decelerative fashion. In contrast, the accelerative phase of the RV is $67.5 \% \pm 4.9 \%$ of its course, significantly shorter than that of the LV $(\mathrm{p}<.001)$. The dP/dt_min of the LV and RV were $-1013.7 \pm 188.1 \mathrm{mmHg} / \mathrm{s}$ and $-402.2 \pm$ $165.3 \mathrm{mmHg} / \mathrm{s}$, respectively $(\mathrm{p}<.0001)$. The ratio of pressure at dP/dt_min to Pmax (P at dP/dt_min / Pmax) was significantly lower in the RV than in the $\mathrm{LV}(28.6 \% \pm 14.8 \%$ and $58.3 \% \pm 7.5 \%$, respectively $; \mathrm{p}<.0001)$.

\section{DIASTOLIC PARAMETERS OF THE LV AND RV IN THE CONTROL GROUP}

The obtained parameter values for $\tau \mathrm{E}, \tau \mathrm{L}$, and the kinematic model parameters Ek and $\mu$ were compared between the $\mathrm{LV}$ and
$\mathrm{RV}$ in the control group to assess the characteristics of normal RV diastolic physiology. Thereafter, the results obtained from $\mathrm{RV}$ pressure in the PAH group were compared with those of the normal RV to elucidate the RV diastolic pathophysiology in pressure overload (Fig. 3). In the control group, $\tau \mathrm{E}$ and $\tau \mathrm{L}$ were not significantly different between the LV and RV (33.1 \pm $6.9 \mathrm{~ms}$ vs. $32.5 \pm 14.6 \mathrm{~ms}$, and $12.6 \pm 2.4 \mathrm{~ms}$ vs. $14.5 \pm 7.2 \mathrm{~ms}$, respectively). Furthermore, $\tau \mathrm{E}$ and $\tau \mathrm{L}$ of the $\mathrm{PAH} \mathrm{RV}$ were 46.8 $\pm 15.5 \mathrm{~ms}$ and $19.6 \pm 6.0 \mathrm{~ms}$, respectively, and they were not significantly different from those of the normal RV. In the analysis of the kinematic model, Ek was significantly lower in the normal $\mathrm{RV}$ than in the normal LV $\left(487.0 \pm 99.6 \mathrm{~s}^{-2} \mathrm{vs} .858 .1 \pm 162.7 \mathrm{~s}^{-2}\right.$, $\mathrm{p}<.0001$ ), whereas $\mu$ was significantly higher in the RV than in the LV $(41.1 \pm 10.4 \mathrm{~ms}$ vs. $21.5 \pm 3.8 \mathrm{~ms}, \mathrm{p}<.0001)$. These results indicate that the normal RV has lower stiffness/elastic recoil and superior cross-bridge relaxation.

\section{DIASTOLIC PARAMETERS OF THE RV IN THE PAH GROUP}

Measured $\tau \mathrm{E}$ and $\tau \mathrm{L}$ were not significantly different between the PAH and Control groups $(46.75 \pm 15.51 \mathrm{~ms}$ vs. $32.5 \pm 14.6 \mathrm{~ms}$, and $19.6 \pm 5.9 \mathrm{~ms}$ vs. $14.5 \pm 7.2 \mathrm{~ms}$, respectively). The PAH group had significantly higher Ek than the control group $(915.9 \pm 84.2$ $\mathrm{s}^{-2}$ vs. $\left.487.0 \pm 99.6 \mathrm{~s}^{-2}, \mathrm{p}<.0001\right)$ and significantly lower $\mu$ than the control group $(16.5 \pm 4.3 \mathrm{~ms}$ vs. $41.1 \pm 10.4 \mathrm{~ms}, \mathrm{p}<.0001)$. These results demonstrate that the PAH RV has higher stiffness/elastic recoil and lower active relaxation in diastole.

\section{ECHOCARDIOGRAPHIC MEASUREMENT}

The conventional echocardiographic parameters that are measured included : E-wave acceleration time (AT), deceleration
A

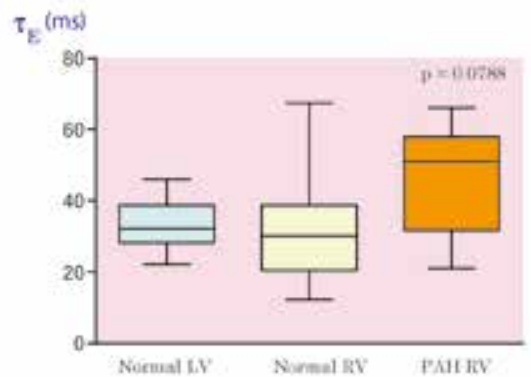

$\mathrm{C}$

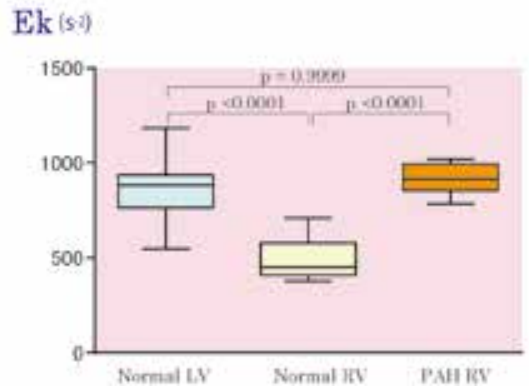

B

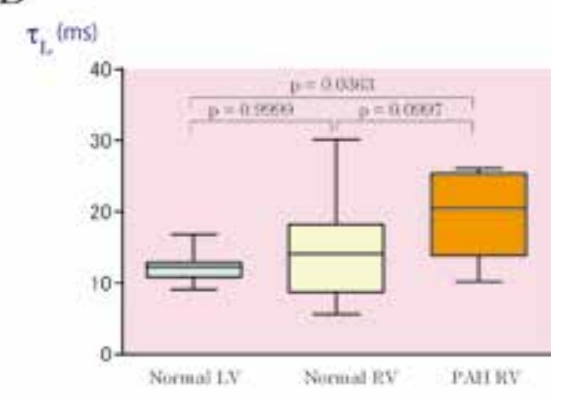

D

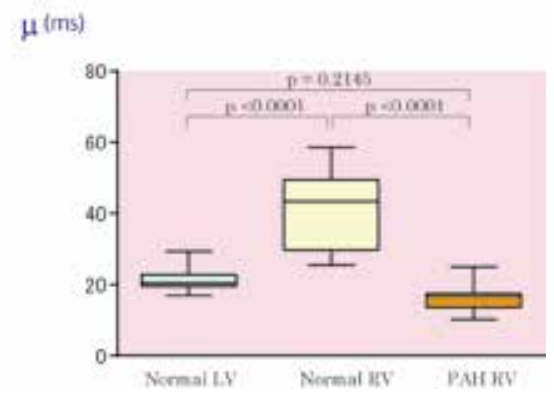

Fig. 3 The values of $\tau$ E, $\tau \mathrm{L}$, Ek, and $\mu$ of the normal $\mathrm{LV}$, normal RV, and RV in the PAH group are shown. Boxes, IQR ; central line, median. Whiskers, 5th-95th percentiles.

n.s., not significant.

Figure modifies from Haybuchi Y, et al. (17) with permission. 
time (DT), peak E- and A-wave velocities (E-peak and A-peak, respectively), and the E/A ratio. Peak early mitral and tricuspid annular velocity $\left(\mathrm{e}^{\prime}\right)$ and peak late annular velocity $\left(\mathrm{a}^{\prime}\right), \mathrm{E} / \mathrm{e}^{\prime}$, and $\mathrm{e}^{\prime} / \mathrm{a}^{\prime}$ are similarly calculated from the Doppler tissue recordings.

\section{DOPPLER E-WAVE ANALYSIS USING KINEMATIC MODEL}

Transmitral and transtricuspid E-waves can be assessed as follows. Using a custom MATLAB release 2015a (MathWorks, Natick, MA, USA) program, beats with clear contours are selected, digitized, and cropped. The maximum velocity envelope is determined from the digitized E-wave image and then used to obtain the automated parametrized diastolic filling (PDF) fit. For the purpose of detecting the appropriate velocity profile, the program searches each time point of the image from the top down for the first pixel having a brightness higher than or equal to a user defined threshold level, matching each time point with a velocity. Furthermore, it is assumed that there must be an upper limit to the velocity difference between two time points a few milliseconds apart. The algorithm accordingly discards a detected velocity that differs too much from the previous velocity. Discarded velocities are displayed for reference and transparency as to the behavior of the algorithm.

The E-waves are then used to compute the PDF parameters. The PDF formalism characterizes the suction-initiated transmitral or transtricuspid flow. This is analogous to the kinematics of a previously displaced, damped, harmonic oscillator after it recoils from a resting state. This methodology utilizes Newton's Second Law, with the predictions of the E-wave contours parametrized on the basis of the chamber stiffness, relaxation/ viscoelasticity, and the load. The equation that describes the balance of forces in a damped harmonic oscillator is :

$$
m \frac{\mathrm{d}^{2} x}{\mathrm{~d} t^{2}}+c \frac{\mathrm{d} x}{\mathrm{~d} t}+k x=0
$$

where $\mathrm{m}(\mathrm{g}), \mathrm{c}(\mathrm{g} / \mathrm{s})$ and $\mathrm{k}\left(\mathrm{g} / \mathrm{s}^{2}\right)$ represent inertia, relaxation (damping) and ventricular stiffness/elastic recoil (spring constant), respectively. The parameter $\mathrm{x} 0(\mathrm{~cm})$ indicates the load and represents the initial displacement of the spring before motion, which corresponds to the elastic strain stored in the myocardium and surrounding structures available at the mitral or tricuspid valve openings that facilitate the mechanical recoil (14-16). When the initial velocity $(\mathrm{dx} / \mathrm{dt})$ of the system is zero, this corresponds to no transmitral or transtricuspid flow prior to the valve opening. The inertial term $\mathrm{m}(\mathrm{g})$ is normalized to 1 in order to enable the computation of $c$ and $k$ per unit mass. These parameters $(\mathrm{k}, \mathrm{c}$, and $\mathrm{x} 0)$ can be directly determined from the clinical E-wave contour. The estimated model parameters are averaged values within the cardiac phase of interest. Expressions describing the velocity of motion as a function of time are derived from the foundational equation (8). For underdamped cases, which are defined by $c^{2}-4 \mathrm{k}<0$, the expression for the velocity (v) as a function of time $(\mathrm{t})$ is

$$
v(t)=\frac{-k \mathrm{x}_{0}}{\omega} \exp (-c \cdot t / 2) \cdot \sin (\omega \cdot t),
$$

where

$$
\omega=\sqrt{4 k-\mathrm{c}^{2}} / 2
$$

For the overdamped cases, which are defined by $c^{2}-4 \mathrm{k}>0$, the expression is

$$
v(t)=\frac{-k \mathrm{x}_{0}}{\beta} \exp (-c \cdot t / 2) \cdot \sin (\beta \cdot t),
$$

$$
\begin{aligned}
& \text { where } \\
& \beta=\sqrt{\mathrm{c}^{2}-4 k} / 2
\end{aligned}
$$

For critically damped cases, which are defined by $c^{2}-4 \mathrm{k}=0$, the expression is

$$
v(t)=-k \mathrm{x}_{0} \cdot t \cdot \exp (-c \cdot t / 2)
$$

The output of the Levenberg-Marquardt algorithm is used to determine the PDF parameter values for $\mathrm{k}$, c, and $\mathrm{x} 0$, while the $\mathrm{E}$-wave maximum velocity envelope is used as the input via a custom LabVIEW 2016 (National Instruments, Austin, TX, USA) interface. The gold-standard methods (simultaneous micromanometric hemodynamics and echocardiography) have been extensively used to validate the physiological interpretation. Results have shown these parameters are causally related to the chamber properties that determine diastolic function (14, 28-30). Physiological conditions can additionally be determined from the damped harmonic oscillator derived parameters such as $\mathrm{kx} 0$, which is the peak force in the spring that corresponds to the peak atrioventricular pressure gradient that generates the E-wave $(14,15) ; 1 / 2 \mathrm{kx}^{2}$, which indicates the stored potential elastic energy that is capable of generating the recoil (14); the peak resistive force (cE-peak), which is the force that resists filling at the peak flow; and $\mathrm{c}^{2}-4 \mathrm{k}$, which indicates the balance between the factors both driving and resisting the ventricular filling (14-16).

We compared the kinematic model parameters $\mathrm{c}, \mathrm{k}$, and $\mathrm{x} 0$ between the LV and the RV in the control group in order to assess the characteristics of the normal RV diastolic physiology. Subsequently, results from the E-waves in the PAH group were compared with those obtained from the normal RV to elucidate the RV diastolic pathophysiology during the RV pressure overload. Figure 4 shows 2 representative examples of transtricuspid Doppler E-wave velocity profile edge detection and fitted curves. These examples demonstrate the method and process on how the digitized E-wave image was used to determine the maximum velocity envelope, from which the automated PDF fit was obtained.

\section{KINEMATIC MODEL-BASED DIASTOLIC PARAME- TERS OF THE RV IN THE PAH GROUP}

As compared to the control group, the PAH group exhibited significantly greater values for the parameters k $(182.5 \pm 72.4 \mathrm{~g} /$ $\mathrm{s}^{2}$ vs. $\left.135.7 \pm 49.5 \mathrm{~g} / \mathrm{s}^{2}, \mathrm{p}=0.0232\right)$ and c $(21.9 \pm 6.5 \mathrm{~g} / \mathrm{s}$ vs. $10.6 \pm$ $5.2 \mathrm{~g} / \mathrm{s}, \mathrm{p}<0.0001)$. These results demonstrate that the PAH RV has a higher stiffness and inferior active relaxation in diastole. The PAH and control groups had an indistinguishable initial load prior to the tricuspid valve opening ( $\mathrm{x}_{0}: 7.7 \pm 2.4 \mathrm{~cm}$ in $\mathrm{PAH} ; 8.2 \pm 2.9 \mathrm{~cm}$ in the control group) (Fig. 5). The PAH group also exhibited greater values of $\mathrm{cE}$-peak, $\mathrm{kx} 0$, and $1 / 2 \mathrm{kx} 0^{2}$.

\section{CORRELATION BETWEEN KINEMATIC PARAME- TERS AND RV PERFORMANCE IN THE PAH GROUP}

In order to analyze the relationships between the kinematic -based parameter values and PAH severity and RV systolic performance, we evaluated the correlation between kinemat- 
ic parameters and RVFAC (right ventricular fractional area change) and TRPG (tricuspid regurgitation peak gradient) (Fig. 6). RVFAC did not have significant correlation with $\mathrm{k}$, c, and $\mathrm{x}$.
Although there was significant correlation between TRPG and $\mathrm{k}$ $(r=0.778, p=0.0008)$, TRPG did not have significant correlation with $\mathrm{c}$ and $\mathrm{x} 0$.
A

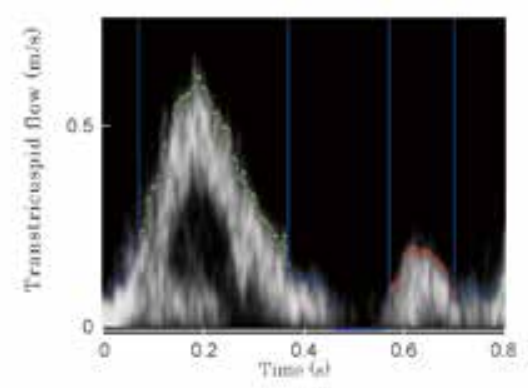

C

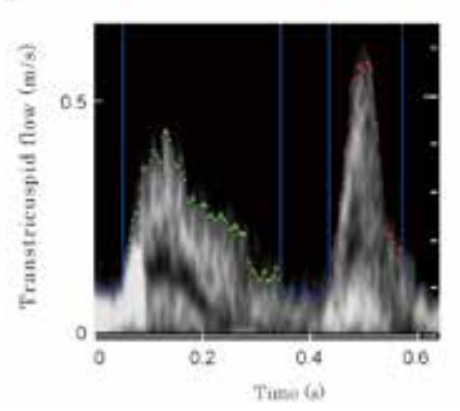

B

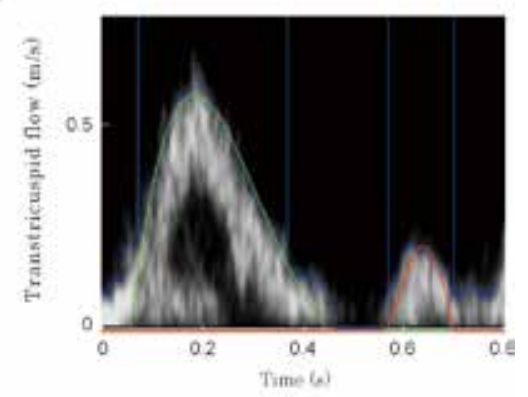

D

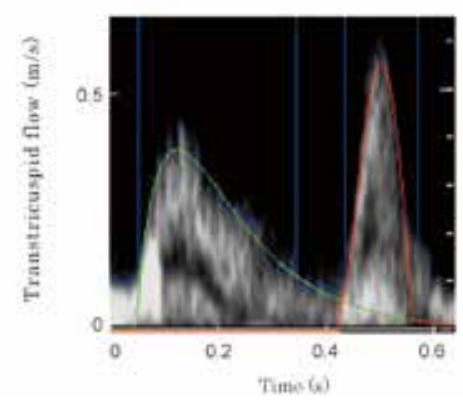

Fig. 4 Quantitation of diastolic function via the PDF formalism.

Representative transtricuspid E-wave Doppler images from a normal subject (A, B) and a PAH patient (C, D) are shown. Doppler E-wave velocity profile edge detection and fitted curves were shown. The digitized E-wave maximum velocity envelope is identified (A, C) and fitted using the Levenberg-Marquardt method by the solution to the PDF model (B, D), which yielded the 3 unique best fit PDF parameters of chamber stiffness $\mathrm{k}$, relaxation c, and initial load x0.

Control subject (A, B) parameters : $\mathrm{x} 0=11.4 \mathrm{~cm}, \mathrm{c}=10.1 \mathrm{~g} / \mathrm{s}, \mathrm{k}=88.6 \mathrm{~g} / \mathrm{s} 2 ;$ MSE $5.3 \mathrm{~cm} 2 / \mathrm{s} 2$.

$\mathrm{PAH}$ patient $(\mathrm{C}, \mathrm{D})$ parameters $\mathrm{x} 0=9.2 \mathrm{~cm}, \mathrm{c}=27.7 \mathrm{~g} / \mathrm{s}, \mathrm{k}=148.1 \mathrm{~g} / \mathrm{s} 2 ;$ MSE $5.1 \mathrm{~cm} 2 / \mathrm{s} 2$.

Figure modifies from Haybuchi Y, et al. (18) with permission.

A

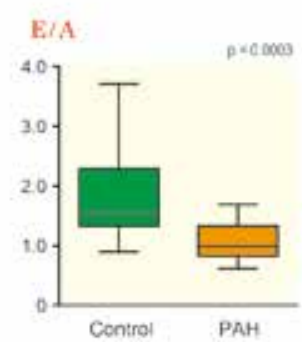

D

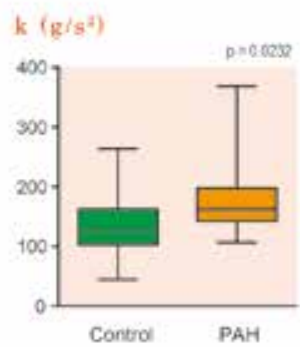

B

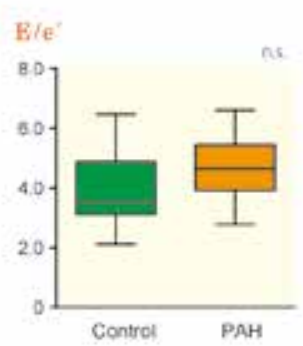

E

$(4:(\mathrm{g} / \mathrm{s})$

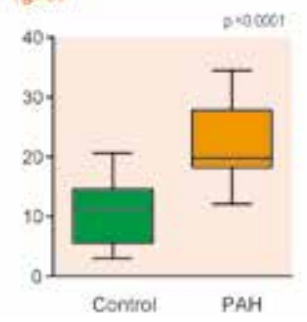

$\mathrm{C}$

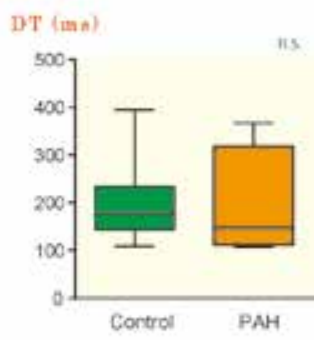

F

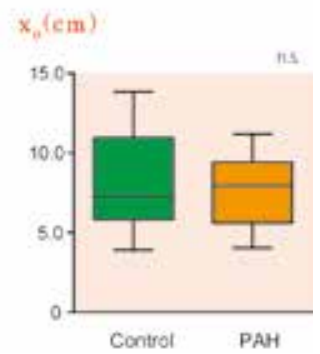

Fig. 5 Conventional and kinematic model-based RV diastolic parameters.

The values of E/A (A), E/e' (B), DT (C), $\mathrm{k}(\mathrm{D}), \mathrm{c}(\mathrm{E})$, and $\mathrm{x}_{0}(\mathrm{~F})$ were compared between the control and the PAH groups. Boxes, IQR ; Central line, median; Whiskers, minimum and maximum.

Figure modifies from Haybuchi Y, et al. (18) with permission. 
A

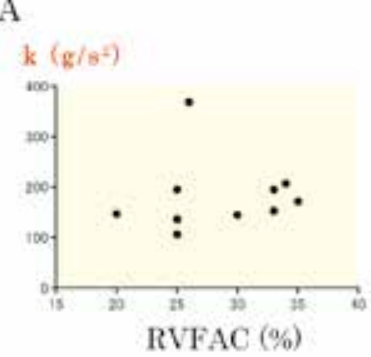

D

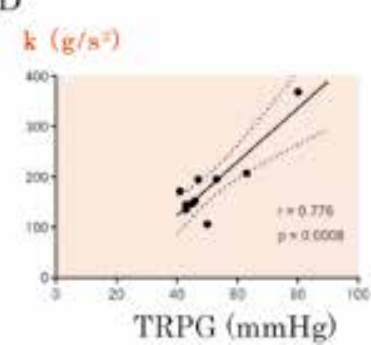

B

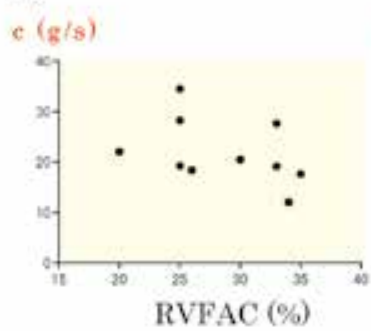

E

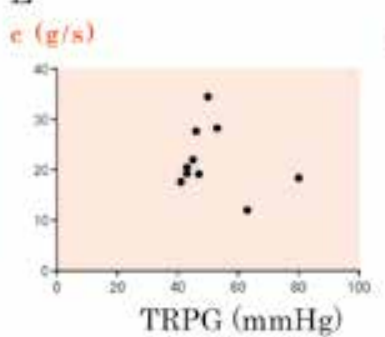

C

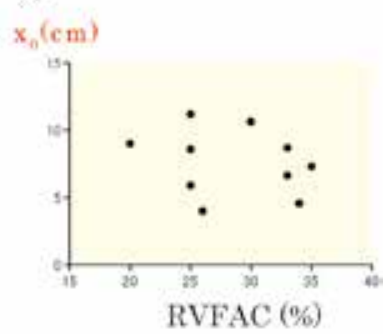

F

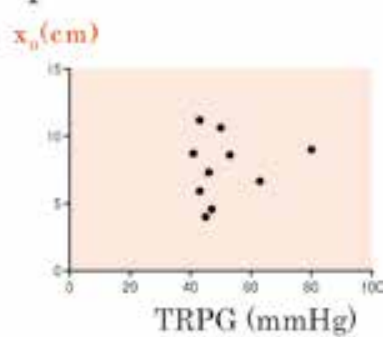

Fig. 6 Correlation between the kinematic model parameters and RV performance in patients with PAH

RVFAC had no significant correlation with $\mathrm{k}(\mathrm{A}), \mathrm{c}(\mathrm{B})$, and $\mathrm{x} 0(\mathrm{C})$. There was significant correlation between TRPG and $\mathrm{k}(\mathrm{D})$, whereas there were no significant correlation with c (E) and $\mathrm{x} 0$ (F). Linear regression lines with the 95\% confidence interval (dashed lines) are indicated. RVFAC, right ventricular fractional area change; TRPG, tricuspid regurgitation peak gradient

Figure modifies from Haybuchi Y, et al. (18) with permission.

\section{ASSESSMENT OF RV DIASTOLIC FUNCTION USING BY KINEMATIC MODEL-BASED ANALYSIS}

Our clinical investigation demonstrates that the causality-based kinematic parameters, Ek and $\mu$, could precisely characterize load-independent RV diastolic function, whereas $\tau \mathrm{E}$ and $\tau \mathrm{L}$ of the RV did not show significant differences between the control and PAH groups. Furthermore, the analysis of the transtricuspid E-waves via the PDF method also generates the parameters of chamber stiffness/elastic recoil and relaxation/ viscosity that is useful for non-invasive diagnosis.

We have shown that the RV has significantly different diastolic properties, including stiffness/elastic recoil and active relaxation, from the LV. The kinematic model demonstrated that the normal RV has lower passive stiffness/restoring and higher active relaxation than the normal LV. Moreover, the RV in the PAH group was significantly stiffer and had slower crossbridge detachment relaxation than the RV in the control group. This method is found to be quite useful way for evaluating RV diastolic dysfunction in PAH patients. Although some previous reports suggest the usefulness of $\tau_{\mathrm{E}}$ and $\tau_{\mathrm{L}}$ for the assessment of RV diastolic dysfunction in PAH patients $(5,9,31)$, this issue has remained controversial. Our results do not show significant differences in these indices between the normal and PAH groups. This discrepancy might result from the subjects' age, disease duration, and severity. The progression of deterioration of RV diastolic function, which consists of active relaxation and stiffness/ elastic recoil, might differ between children and adults.

Furthermore, the RV and LV pressure decreases were found to follow distinct time courses. The initial accelerative phase until $\mathrm{dP} / \mathrm{dt}$ _min is relatively longer, and the subsequent decelerative phase is shorter in the RV than in the LV. In this respect, the RV time constants $\tau \mathrm{E}$ and $\tau \mathrm{L}$ evaluate a quite short segment of RV pressure decay. However, on the molecular level, both $\tau \mathrm{E}$ and $\tau \mathrm{L}$ have been shown to correlate with active relaxation as defined by deactivation events, such as cross-bridge cycling, cal- cium handing, or lusitropism $(32,33)$, but neither can fully characterize the full range of the IVR pressure decrease. We should, therefore, be aware that the RV time constant only evaluates a minor portion of the RV pressure fall and has low reproducibility. When compared with $\tau \mathrm{E}$ and $\tau \mathrm{L}$ models, the kinematic model parameters Ek and $\mu$ provides a superior fit to IVR pressure and higher reproducibility.

Previous work by Chung and Kovács demonstrated that IVR pressure is precisely determined by the interplay of stiffness/ elastic recoil and damping/relaxation forces (25). The relative contributions of stiffness and relaxation to IVR pressure decay are characterized by the stiffness/restoring parameter Ek and the damping/relaxation parameter $\mu$. This kinematic model successfully unifies the previously unrelated $\tau \mathrm{E}$ and $\tau \mathrm{L}$ models of isovolumic pressure decay in a parametric limit sense. The model proposed in Eq. 3B explains why PPP contours can change shape. A linear IVR PPP segment is one where the relaxation parameter $(1 / \mu)$ is large compared with the elastic term (Ek) (31). As the elastic term increases, the IVR PPP segment becomes more curvilinear (31). A recent study involving humans demonstrated RV hypertrophy with collagen deposition, increased sarcomeric stiffness, and changed titin isoform and phosphorylation $(3,32)$. RV diastolic behavior should be evaluated from the perspectives of stiffness and relaxation. In this respect, the kinematic model established the parameters conforming to the pathophysiological state.

The load dependence of $\tau \mathrm{E}$ and $\tau \mathrm{L}$ is well established (33-35), and, therefore, the variations in $\tau \mathrm{E}$ and $\tau \mathrm{L}$ between subjects may be the result of intrinsic chamber property differences or may be caused by extrinsic load effects in the assessment of $\mathrm{LV}$ diastolic function. We had shown that RV $\tau \mathrm{E}$ and $\tau \mathrm{L}$ are significantly correlated with RVEDP (17). Thus, a load-independent index that overcomes the limitations of $\tau \mathrm{E}$ and $\tau \mathrm{L}$ would be advantageous. The kinematic parameters Ek and $\mu$ were relatively independent of preload (17). Furthermore, Shmuylovich and Kovács applied this kinematic model and derived a load-independent parameter, 
named MLIIIVPD, which is the constant slope between the effective peak elastic recoil forces that drive pressure decline during isovolumic relaxation and the peak resistive forces that oppose cross-bridge uncoupling and pressure decline (36).

With the combined pressure conductance catheter, it has become possible to determine ventricular pressure and volume simultaneously. The gold standard for measuring load-independent diastolic stiffness by pressure-volume (PV) analysis is not without risk in PAH patients because it requires temporal preload reduction $(37,38)$. In left heart failure, this is circumvented by the development of single-beat analyses of the diastolic PV relationship $(39,40)$. However, it is unclear whether this analysis could also be used for the RV in PAH. Furthermore, since precise $\mathrm{RV}$ volumetric measurement is challenging, it would be quite difficult to assess RV diastolic function using PV analysis.

In the echocardiographic study, the parameter $\mathrm{k}$ represents the chamber stiffness/elastic recoil property. As described above, chamber stiffness $(\mathrm{dP} / \mathrm{dV})$ as evaluated by invasive cardiac catheterization, has been shown to be linearly related to the spring constant $\mathrm{k}\left(\mathrm{g} / \mathrm{s}^{2}\right)(28-30)$. It has been shown that PDF analysis of the Doppler E-wave can accurately determine the LV diastatic passive chamber stiffness (41). The higher $\mathrm{k}$ value for the RV in the PAH group is consistent with an elevated RV filling pressure.

Kinematically, the lumped viscoelastic (resistive) properties of the system are represented by the c parameter. Thus, any source of energy loss that opposes motion during the filling are considered to be a part of the physiological analog. Increased values of $c$ can be manifested by various factors that can influence filling via an energy loss. These factors can include blood viscosity, delayed relaxation, dynamic friction during sarcomere lengthening that occurs as the detached myosin heads slide past the thin filaments, pericardial effects, and the viscosity of the extracellular matrix (42-44). Changes in intracellular calcium handling and high myofilament calcium sensitivity in PAH have been reported to impair proper relaxation of cardiomyocytes (2). Moreover, these pathological conditions are known to affect the kinematic model parameters.

The $\mathrm{x} 0$ value is related to the load responsible for compressing the elastic myocardium at the end of systole, which is a prerequisite in order for the restoring force to arise. The $\mathrm{x} 0$ value is also closely related to the velocity time integral (VTI) of the E-wave. In normal subjects, the parameter xo was lower in the RV versus the LV. This might be because of the difference of the area at the tip of the valve leaflets found between them. Furthermore, this could possibly be due to the difference of the E/A ratio, Doppler beam angle, and respiratory condition present at the time of the recording. There was no significant difference in the volumetric preload parameter xo between the control and PAH groups. This value is specifically determined by several factors, including stroke volume, volumetric E/A ratio, RV filling pressure, and right atrial pressure. This finding suggests that during pathological conditions, these PAH patients maintained the stroke volume while at rest.

The initial maximum recoil force $(\mathrm{kx} 0)$ and the stored potential elastic energy $\left(1 / 2 \mathrm{kx}^{2}\right)$ were significantly higher in the $\mathrm{PAH}$ versus the control group. The product $\mathrm{kx}$, which is analogous to the maximum atrioventricular pressure gradient that generates the E-wave by mechanical suction, represents the initial peak force in the spring. The results of a previous investigation that found the kxo was more consistent than the modified Bernoulli equation $\left(\mathrm{PG}=4 \mathrm{~V}^{2} ; \mathrm{PG}\right.$, pressure gradient $; \mathrm{V}$, blood flow velocity) with regard to predicting the instantaneous maximum pressure gradient also validates the above predictions (15).

Kinematically, the potential energy in the spring prior to its release is represented by $1 / 2 \mathrm{kx}^{2}$. The physiological analog for this factor is the stored elastic strain energy that is avail- able at the tricuspid valve opening. This energy generates the chamber recoil, which leads to the generation of the E-wave. The significantly greater values for $\mathrm{kx} 0$ and $1 / 2 \mathrm{kx}^{2}$ in the $\mathrm{PAH}$ group suggest that an adaptive mechanism via the hypertrophic $\mathrm{RV}$ chamber is required in order to maintain a stroke volume. This is shown by our results that indicated that there was no significant difference for the load $\mathrm{x} 0$ between the control and PAH groups. However, this occurs at the cost of increased energy utilization. In addition, our data also indicated that the cE-peak was significantly higher in the PAH patients versus the control subjects, which indicates a higher resistance (impaired relaxation) to the RV filling. A greater atrioventricular pressure gradient $\mathrm{kx} 0$ (and consequently, $1 / 2 \mathrm{kx} 0^{2}$ ) would be expected to be generated in response to increased damping (resistive losses) of the transtricuspid flow, as is manifested by greater values of c and cE-peak, in order to maintain the load xo or stroke volume.

Although previous reports and the ASE guidelines have suggested the usefulness of conventional parameters in the assessment of RV diastolic dysfunction $(9,31,46,47)$, the utilization of these parameters has remained controversial $(31,46,47)$. The present study revealed that $\mathrm{E} / \mathrm{e}^{\prime}$ and DT of the transtricuspid E-wave did not exhibit any significant differences between the control and PAH groups, whereas there was a significant difference for the E/A. This discrepancy regarding the usefulness of the conventional indexes might be associated with the subjects' age, disease duration, and pathological severity. Moreover, the progression of the RV diastolic function deterioration, which consists of active relaxation and stiffness/elastic recoil, might differ between children and adults. In most of the studies that have examined these differences, results indicated there was a modest correlation between the $\mathrm{E} / \mathrm{A}$ ratio and increasing age (48, 49). Since the peak velocity of E-wave increases during inspiration, this causes an increase in the E/A ratio. Furthermore, while increases in the E-peak are caused by tachycardia, a relatively greater increase in the A-peak will result in a decrease in the E/A ratio $(49,50)$. These parameters are also sensitive to changes in preload. Thus, while a reduction in the preload will lead to a decrease in $\mathrm{E}$, there will be a relatively smaller decrease in $\mathrm{A}$, thereby causing the E/A to decrease (51-53). However, it should be noted that since the conventional echocardiographic diastolic function indexes are empirical, these values will not provide any mechanistic information on the chamber property, e.g., stiffness and relaxation. Moreover, since E-wave parameters are not derived from basic physiologic principles that govern filling, these parameters are considered to be load dependent (54-56). In addition, the complex interplay of simultaneous physiologic determinants and chamber properties are responsible for generating these indexes. Moreover, a previous study has demonstrated that E-wave DT was specifically dependent upon both the chamber stiffness and the chamber relaxation/viscoelasticity (57).

All the global physiologic determinants of the contour were accounted for by the 3 lumped parameters c, k, and xo. RV diastolic dysfunction determines ventricular performance and patient outcomes for many conditions. Moreover, this dysfunction may precede the apparent systolic dysfunction (31, 45, 58, 59). Overall, we believe that our current findings indicate that there are great clinical implications for this method with regard to the management of $\mathrm{PAH}$ patients.

\section{CONCLUSION}

This review artilce indicated the validation of kinematic model parameters for assessing RV diastolic function in PAH patients. The PAH patients have higher stiffness/restoring and lower cross-bridge relaxation than the Control group. 


\section{DISCLOSURE}

The authors declare that they have no conflicts of interest.

\section{REFERENCES}

1. Marwick TH, Gillebert TC, Aurigemma G, Chirinos J, Derumeaux G, Galderisi M, Gottdiener J, Haluska B, Ofili E, Segers P, Senior R, Tapp RJ, Zamorano JL: Recommendations on the use of echocardiography in adult hypertension : A report from the European Association of Cardiovascular Imaging (EACVI) and the American Society of Echocardiography (ASE). Eur Heart J Cardiovasc Imaging 16 : 577-605, 2015

2. Rain S, Andersen S, Najafi A, Gammelgaard Schultz J, da Silva Gonçalves Bós D, Handoko ML, Bogaard HJ, Vonk-Noordegraaf A, Andersen A, van der Velden J, Ottenheijm CA, de Man FS : Right ventricular myocardial stiffness in experimental pulmonary arterial hypertension : relative contribution of fibrosis and myofibril stiffness. Circ Heart Fail 9 : e002636, 2016

3. Murch SD, La Gerche A, Roberts TJ, Prior DL, MacIsaac AI, Burns AT : Abnormal right ventricular relaxation in pulmonary hypertension. Pulm Circ 5 : 370-375, 2015

4. van de Veerdonk MC, Kind T, Marcus JT, Mauritz G-J, Heymans MW, Bogaard H-J, Boonstra A, Marques KMJ, Westerhof N, Vonk-Noordegraaf A : Progressive right ventricular dysfunction in patients with pulmonary arterial hypertension responding to therapy. J Am Coll Cardiol. $58: 2511-2519,2011$

5. Frangogiannis NG : Fibroblasts and the extracellular matrix in right ventricular disease. Cardiovasc Res. 113 : 14531464, 2017

6. D'Alonzo GE, Barst RJ, Ayres SM, Bergofsky EH, Brundage BH, Detre KM, Fishman AP, Goldring RM, Groves BM, Kernis JT, Levy PS, Pietra GG, Reid LM, Reeves JT, Rich $\mathrm{S}$, Vreim CE, Williams GW, Wu M : Survival in patients with primary pulmonary hypertension : results from a national prospective registry. Ann Intern Med. 115 : 343-349, 1991

7. Shiina Y, Funabashi N, Lee K, Daimon M, Sekine T, Kawakubo M, Takahashi M, Yajima R, Tanabe N, Kuriyama T, Komuro I : Right atrium contractility and right ventricular diastolic function assessed by pulsed tissue Doppler imaging can predict brain natriuretic peptide in adults with acquired pulmonary hypertension. Int J Cardiol. 135 : 53-59, 2009

8. Kassem E, Humpl T, Friedberg MK : Prognostic significance of 2-dimensional M-mode, and Doppler echo indices of right ventricular function in children with pulmonary arterial hypertension. Am Heart J. 165 : 1024-1031, 2013

9. Okumura K, Slorach C, Mroczek D, Dragulescu A, Mertens L, Redington AN, Friedberg MK : Right ventricular diastolic performance in children with pulmonary arterial hypertension associated with congenital heart disease : correlation of echocardiographic parameters with invasive reference standards by high-fidelity micromanometer catheter. Circ Cardiovasc Imaging. 7 : 491-501, 2014

10. Weiss J, Frederiksen JW, Weisfeldt ML : Hemodynamic determinants of the time course of fall in canine left ventricular pressure. J Clin Invest. 58: 751-760, 1976

11. Kass DA : Assessment of diastolic dysfunction : invasive modalities. Cardiol Clin. 18:571-586, 2000

12. Correia-Pinto J, Henriques-Coelho T, Magalhães S, Leite-Moreira AF : Pattern of right ventricular pressure fall and its modulation by afterload. Physiol Res. $53: 19-26,2004$

13. Cosson S, Kevorkian JP : Left ventricular diastolic dysfunction : an early sign of diabetic cardiomyopathy? Diabetes Metab 29 : 455-466, 2003

14. Kovács SJ Jr., Barzilai B, Perez JE : Evaluation of diastolic function with Doppler echocardiography : the PDF formalism. Am J Physiol 252 : H178-187, 1987

15. Bauman L, Chung CS, Karamanoglu M, Kovács SJ : The peak atrioventricular pressure gradient to transmitral flow relation : kinematic model prediction with in vivo validation. J Am Soc Echocardiogr 17 : 839-844, 2004

16. Shmuylovich L, Kovács SJ : Load-independent index of diastolic filling : model-based derivation with in vivo validation in control and diastolic dysfunction subjects. J Appl Physiol (1985) $101:$ 92-101, 2006

17. Hayabuchi Y, Ono A, Homma Y, Kagami S : Analysis of Right Ventricular Myocardial Stiffness and Relaxation Components in Children and Adolescents With Pulmonary Arterial Hypertension. J Am Heart Assoc. 19 ; 7(9). pii : e008670, 2018

18. Hayabuchi Y, Homma Y, Kagami S : Right Ventricular myocardial stiffness and relaxation components by kinematic model-based transtricuspid flow analysis in children and adolescents with pulmonary arterial hypertension. Ultrasound Med Biol. 45 : 1999-2009, 2019

19. Eucker SA, Lisuaskas JB, Singh J, Kovács SJ Jr. : Phase plane analysis of left ventricular hemodynamics. $J$ Appl Physiol (1985). 90 : 2238-2244, 2001

20. Yellin EL, Hori M, Yoran C, Sonnenblick EH, Gabbay S, Frater RW : Left ventricular relaxation in the filling and nonfilling intact canine heart. Am J Physiol Heart Circ Physiol. 250 : H620-H629, 1986

21. Matsubara H, Takaki M, Yasuhara S, Arki J, Suga H : Logistic time constant of isovolumic relaxation pressure-time curve in the canine left ventricle. Circulation. $92: 2318-2326$, 1995

22. Miki S, Murakami T, Iwase T, Tomita T, Nakamura Y, Kawai C : Doppler echocardiographic transmitral peak early velocity does not directly reflect hemodynamic changes in humans : importance of normalization to mitral stroke volume. J Am Coll Cardiol. 17 : 1507-1516, 1991

23. Cheng C, Igarashi Y, Little W : Mechanism of augmented rate of left ventricular filling during exercise. Circ Res. $70: 9-19,1992$

24. Press WH : Numerical Recipes $3^{\text {rd }}$ edition : The Art of Scientific Computing. New York : Cambridge University Press, 2007.

25. Chung CS, Kovács SJ : Physical determinants of left ventricular isovolumic pressure decline: model prediction with in vivo validation. Am J Physiol Heart Circ Physiol. 294 : H1589-H1596, 2008

26. Feynman RP, Leighton RB, Sands M : The Feynman Lectures on Physics, boxed set: The new millennium edition. Addison-Wesley, 2011.

27. Bernardi L, Uretsky BF, Reddy PS, Boudreau R : Modeling the isovolumic relaxation period. Cathet Cardiovasc Diagn. $11: 255-268,1985$

28. Kovács SJ, Setser R, Hall AF : Left ventricular chamber stiffness from model-based image processing of transmitral Doppler E-waves. Coron Artery Dis 8 : 179-187, 1997

29. Kovács SJ, Meisner JS, Yellin EL : Modeling of diastole. Cardiol Clin 18: 459-487., 2000

30. Lisauskas JB, Singh J, Bowman AW, Kovács SJ : Chamber properties from transmitral flow : prediction of average and passive left ventricular diastolic stiffness. J Appl Physiol (1985) $91: 154-162,2001$ 
31. Leeuwenburgh BP, Steendijk P, Helbing WA, Baan J : Indexes of diastolic RV function : load dependence and changes after chronic RV pressure overload in lambs. Am J Physiol Heart Circ Physiol. 282 : H1350-1358, 2002

32. Camacho SA, Brandes R, Figueredo VM, Weiner MW : Ca2+ transient decline and myocardial relaxation are slowed during low flow ischemia in rat hearts. J Clin Invest. 93 : 951957, 1994

33. Mizuno J, Araki J, Mikane T, Mohri S, Imaoka T, Matsubara H, Okuyama H, Kurihara S, Ohe T, Hirakawa M, Suga $\mathrm{H}$ : Logistic time constant of isometric relaxation force curve of ferret ventricular papillary muscle : reliable index of lusitropism. Jpn J Physiol. 50 : 479-487, 2000

34. Hori M, Inoue M, Kitakaze M, Tsujioka K, Ishida Y, Fukunami M, Nakajima S, Kitabatake A, Abe H: Loading sequence is a major determinant of afterload-dependent relaxation in intact canine heart. Am J Physiol Heart Circ Physiol. 249: H747-H754, 1985

35. Zile MR, Gaasch WH. : Load-dependent left ventricular relaxation in conscious dogs. Am J Physiol Heart Circ Physiol. 261 : H691-H699, 1991

36. Shmuylovich L, Kovács SJ : Stiffness and relaxation components of the exponential and logistic time constants may be used to derive a load-independent index of isovolumic pressure decay. Am J Physiol Heart Circ Physiol.295 : H2551-H2559, 2008

37. Senzaki H, Kass DA : Analysis of isovolumic relaxation in failing hearts by monoexponential time constants overestimates lusitropic change and load dependence : mechanisms and advantages of alternative logistic fit. Circ Heart Fail. $3: 268-276,2010$

38. Westerhof N, Stergiopulos N, Noble MIM : Snapshots of hemodynamics : an aid for clinical research and graduate education. 2nd ed. New York, NY : Springer ; 2010.

39. Klotz S, Hay I, Dickstein ML, Yi GH, Wang J, Maurer MS, Kass DA, Burkhoff D : Single-beat estimation of end-diastolic pressure-volume relationship : a novel method with potential for noninvasive application. Am J Physiol Heart Circ Physiol. 291 : H403-H412, 2006

40. Burkhoff D, Mirsky I, Suga H : Assessment of systolic and diastolic ventricular properties via pressure-volume analysis : a guide for clinical, translational, and basic researchers. Am J Physiol Heart Circ Physiol. 289 : H501-H512, 2005

41. Mossahebi S, Kovács SJ : Kinematic modeling-based left ventricular diastatic (passive) chamber stiffness determination with in-vivo validation. Ann Biomed Eng 40 : 987-995, 2012

42. Dent CL, Bowman AW, Scott MJ, Allen JS, Lisauskas JB, Janif M, Wickline SA, Kovács SJ : Echocardiographic characterization of fundamental mechanisms of abnormal diastolic filling in diabetic rats with a parameterized diastolic filling formalism. J Am Soc Echocardiogr 14 : 1166-1172, 2001

43. Kass DA : Getting better without AGE : New insights into the diabetic heart. Circ Res 92 : 704 -706, 2003.

44. Chung CS, Methawasin M, Nelson OL, Radke MH, Hidalgo CG, Gotthardt M, Granzier HL : Titin based viscosity in ventricular physiology : an integrative investigation of PEVK-actin interactions. J Mol Cell Cardiol 51 : 428-434, 2011

45. Rudski LG, Lai WW, Afilalo J, Hua L, Handschumacher MD, Chandrasekaran K, Solomon SD, Louie EK, Schiller NB: Guidelines for the echocardiographic assessment of the right heart in adults : a report from the American Society of Echocardiography endorsed by the European Association of Echocardiography, a registered branch of the European Society of Cardiology, and the Canadian Society of Echocardiography. J Am Soc Echocardiogr 23 : 685-713, 2010

46. Sade LE, Gulmez O, Eroglu S, Sezgin A, Muderrisoglu H : Noninvasive estimation of right ventricular filling pressure by ratio of early tricuspid inflow to annular diastolic velocity in patients with and without recent cardiac surgery. $J \mathrm{Am}$ Soc Echocardiogr 20 : 982-988, 2007

47. Sundereswaran L, Nagueh SF, Vardan S, Middleton KJ, Zoghbi WA, Quiñones MA, Torre-Amione G : Estimation of left and right ventricular filling pressures after heart transplantation by tissue Doppler imaging. Am J Cardiol 82 : 352357, 1998

48. Innelli P, Esposito R, Olibet M, Nistri S, Galderisi M: The impact of ageing on right ventricular longitudinal function in healthy subjects : a pulsed tissue Doppler study. Eur J Echocardiogr $10: 491-8,2009$

49. Zoghbi WA, Habib GB, Quinones MA : Doppler assessment of right ventricular filling in a normal population. Comparison with left ventricular filling dynamics. Circulation $82: 1316-1324,1990$

50. Yu CM, Lin H, Ho PC, Yang H : Assessment of left and right ventricular systolic and diastolic synchronicity in normal subjects by tissue Doppler echocardiography and the effects of age and heart rate. Echocardiography $20: 19-27,2003$

51. Guazzi M, Maltagliati A, Tamborini G, Celeste F, Pepi M, Muratori M, Berti M, Guazzi MD : How the left and right sides of the heart, as well as pulmonary venous drainage, adapt to an increasing degree of head-up tilting in hypertrophic cardiomyopathy : differences from the normal heart. $J$ Am Coll Cardiol 36 : 185-193, 2000

52. O'Sullivan CA, Duncan A, Daly C, Li W, Oldershaw P, Henein MY : Dobutamine stress-induced ischemic right ventricular dysfunction and its relation to cardiac output in patients with three-vessel coronary artery disease with angina-like symptoms. Am J Cardiol 96 : 622-627, 2005

53. Pelà G, Regolisti G, Coghi P, Cabassi A, Basile A, Cavatorta A, Manca C, Borghetti A : Effects of the reduction of preload on left and right ventricular myocardial velocities analyzed by Doppler tissue echocardiography in healthy subjects. Eur J Echocardiogr 5 : 262-271, 2004

54. King GJ, Murphy RT, Almuntaser I, Bennett K, Ho E, Brown AS : Alterations in myocardial stiffness in elite athletes assessed by a new Doppler index. Heart 94 : 1323-1325, 2008

55. Paelinck BP, van Eck JW, De Hert SG, Gillebert TC : Effects of postural changes on cardiac function in healthy subjects. Eur J Echocardiogr 4 : 196-201, 2003

56. Pepi M, Guazzi M, Maltagliati A, Berna G, Tamborini $\mathrm{G}$ : Diastolic ventricular interaction in normal and dilated heart during head-up tilting. Clin Cardiol 23 : 665-672, 2000

57. Shmuylovich L, Kovács SJ : E-wave deceleration time may not provide an accurate determination of LV chamber stiffness if LV relaxation/viscoelasticity is unknown. Am J Physiol Heart Circ Physiol 292 : H2712-2720, 2007

58. Dernellis $\mathrm{J}:$ Right atrial function in hypertensive patients : effects of antihypertensive therapy. J Hum Hypertens 15 : 463-470, 2001

59. Gan CT, Holverda S, Marcus JT, Paulus WJ, Marques KM, Bronzwaer JG, Twisk JW, Boonstra A, Postmus PE, Vonk-Noordegraaf A : Right ventricular diastolic dysfunction and the acute effects of sildenafil in pulmonary hypertension patients. Chest $132: 11-17,2007$ 